\title{
CrystEngComm
}

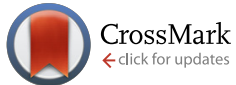

Cite this: CrystEngComm, 2015, 17, 7684

Received 14th August 2015,

Accepted 3rd September 2015

DOI: $10.1039 / c 5 c e 01644 d$

www.rsc.org/crystengcomm

\section{Crystalline adducts of the Lawsone molecule (2-hydroxy-1,4-naphthaquinone): optical properties and computational modelling $\dagger$}

\author{
Anuradha Pallipurath, $\$ \S^{a}$ Jonathan M. Skelton, $+q^{\star a}$ Amit Delori, $\|^{\star a}$ Connor Duffy, ${ }^{b}$ \\ Andrea Erxleben ${ }^{\mathrm{b}}$ and William Jones ${ }^{\mathrm{a}}$
}

\begin{abstract}
Four new crystalline adducts of the Lawsone molecule (2-hydroxy-1,4-naphthaquinone) with 4,4'bipyridine, 4-(2-pyridine-4-ethyl)pyridine, 1,3-di(4-pyridyl)propane and 2-hydroxy pyridine are reported. Adduct formation leads to colour shifts, which are characterised by UV/visible spectroscopy. Complementary quantum-chemical calculations are used to study the energetics of the adduct formation, and to gain insight into the origin of the observed colour changes.
\end{abstract}

\section{Introduction}

The naturally-occurring compound Lawsone (2-hydroxy-1,4naphthoquinone) has been in use for the past 5000 years for utilities ranging from traditional tattoo ink (henna) to medicinal remedies. Since it is an abundant naturally-occurring dye, it is used as artificial-tanning and hair colourants. Its UVabsorbing properties also make it a good anti-tanning agent. ${ }^{1,2}$ Lawsone has also been identified as having anti-fungal, $^{3}$ anti-corrosion ${ }^{4}$ and even anti-cancer properties, ${ }^{5}$ and has been intercalated into $\mathrm{Zn}$ hydroxides to act as a drug-delivery system. ${ }^{6}$ It reacts with primary amino acids to form photoluminescent products, which can be used to detect fingerprints. ${ }^{7}$

In 2005, Todkary et al. reported the existence of two polymorphic forms of Lawsone, formed through solvent interactions. ${ }^{8}$

\footnotetext{
${ }^{a}$ Department of Chemistry, University of Cambridge, Cambridge, CB2 1EW, UK

${ }^{b}$ School of Chemistry, National University of Ireland, Galway, Ireland

$\dagger$ Electronic supplementary information (ESI) available: Contains additional tables and figures to support the discussion in the text. The structures of the reported crystalline adducts 1a-1d are available free of charge from the CCDC under the codes 1418634-1418637 from www.ccdc.cam.ac.uk/getstructures. The PBEsol-optimised gas-phase structures of $\mathbf{1}$, coformers a-d, the $\mathbf{d}_{2}$ dimer, and 1a-1d, as well as the optimised crystal structures of 1a-1d, are available online under the DOI 10.15125/BATH-00137 (http://dx.doi.org/10.15125/BATH-00137). All other experimental characterisation and simulation results are presented in the manuscript and supporting information, with raw data available from the authors on request. For ESI and crystallographic data in CIF or other electronic format see DOI: 10.1039/c5ce01644d

$\$$ These authors contributed equally.

$\S$ Present address: School of Chemistry, National University of Ireland, Galway, Ireland.

ף Present address: Department of Chemistry, University of Bath, Claverton Down, Bath, BA2 7AY, UK. E-mail: jms70@bath.ac.uk

|| Present address: Strathclyde Institute of Pharmacy and Biomedical Sciences, University of Strathclyde, 161 Cathedral Street, Glasgow, G4 0RE, UK. E-mail amitdelori@gmail.com
}

They also observed intrinsic radical formation in both polymorphs, arising from electron-density percolation through intermolecular hydrogen bonds, using electron paramagnetic resonance (EPR) spectroscopy. In contrast, recently-reported cocrystals of Lawsone with tris(hydroxymethyl)aminomethane were found to be EPR inactive. ${ }^{9}$

Due to its $\mathrm{C}=\mathrm{O}$ and $\mathrm{O}-\mathrm{H}$ functional groups, Lawsone readily forms Michael-addition products and Mannich bases, ${ }^{10}$ some of which have antimalarial properties. ${ }^{11,12}$ These functional groups also make the molecule an ideal candidate for forming cocrystals though molecular recognition mediated by $\mathrm{H}$-bonding interactions.

Cocrystallisation reactions are known for their simplicity and versatility. ${ }^{13-15}$ They have found applications in various fields to modify phase-transition temperatures, ${ }^{16}$ to improve solubility, ${ }^{17-22}$ to enhance fluorescence, ${ }^{23-29}$ and to alter melting points. ${ }^{19,30}$ These reactions also find application in the pharmaceutical industry, e.g. for improving the tableting properties,${ }^{31,32}$ dissolution rates ${ }^{33-35}$ and stability ${ }^{36,37}$ of pharmaceutical compounds. They can also be used to improve the elastic properties of materials. ${ }^{38}$

Recently, it has been demonstrated that cocrystallisation can also be used for colour tuning. Yan et al. used cocrystallisation to tune the colour and fluorescence properties of the well-known fluorecscent whitener 1,4-bis-p-cyanostyrylbenzene. ${ }^{24}$ Research by Bučar et al. similarly demonstrated that the colours of pigments can be drastically changed using cocrystallization. ${ }^{26}$ Jones et al. studied the role of proton transfer and disorder in the formation of coloured adducts, ${ }^{39}$ and in their systems found that disorder favoured the formation of coloured adducts, whereas proton transfer disfavoured it. MacGillivray et al. reported a coloured cocrystal between acetaminophen and 2,4-pyridinedicarboxylic acid,$^{40}$ both of which are themselves colourless. 
In this paper, we report four crystalline adducts of Lawsone $(1 ; 1 \mathrm{a}-1 \mathrm{~d})$ with aza-donors, viz. 4,4'-bipyridine (a), 4-(2-pyridine-4-ethyl)pyridine (b), 1,3-di(4-pyridyl)propane (c) and 2-hydroxy pyridine (d; Chart 1). The solid-state structures of the adducts are obtained from single-crystal X-ray diffraction (XRD), and the crystals are further characterised by optical microscopy, thermal analysis, near-infrared (NIR) spectroscopy and UV/visible reflectance. All four crystalline adducts exhibit changes in colour with respect to neat Lawsone.

We have also carried out complementary densityfunctional theory calculations to model the energetics of the adduct formation, and to investigate the origin of the colour changes in terms of the electronic structure.

\section{Experimental}

\section{Synthesis}

Lawsone (2-hydroxy-1,4-naphthoquinone), 4,4'-bipyridine, 4-(2-pyridine-4-ethyl)pyridine, 1,3-di(4-pyridyl)propane and 2-hydroxy pyridine were purchased from Sigma Aldrich and used without further purification. Crystalline adducts 1a-1c were formed by dissolving Lawsone and the coformer in methanol in a 2:1 molar ratio and allowing them to crystallise. Owing to its higher solubility, 1c tended to crystallise only at low temperatures (usually below $4{ }^{\circ} \mathrm{C}$ ), and typically re-dissolved if left standing at room temperature for too long. 1d was obtained by dissolving Lawsone and 2-hydroxy pyridine in dichloromethane in a 1:1 molar ratio, with toluene being added to the mixture to reduce the rate of evaporation.

\section{Spectroscopy}

UV-visible absorption spectra were recorded in a 2:1 mixture of acetonitrile and methanol (to keep the hydrogen-bonded

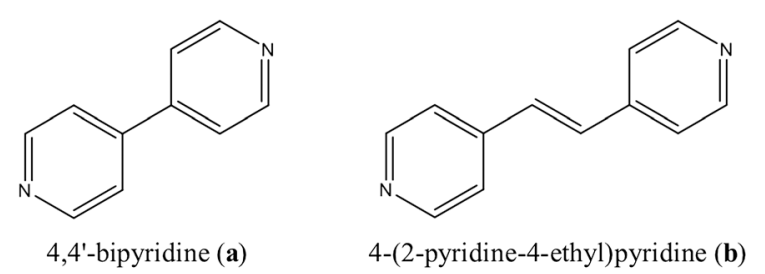<smiles>O=C1C=C(O)C(=O)c2ccccc21</smiles>

2-hydroxy-1,4-naphthaquinone (Lawsone; 1)<smiles>c1cc(CCCc2ccncc2)ccn1</smiles>

1,3-di(4-pyridyl)propane (c)

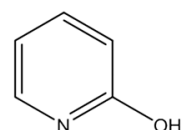

2-hydroxy pyridine (d)
Chart 1 Structures of Lawsone (1), 4,4'-bipyridine (a), 4-(2-pyridine-4ethyl)pyridine (b), 1,3-di(4-pyridyl)propane (c) and 2-hydroxy pyridine (d). species associated) using a Cary 60 Spectrophotometer (Agilent). Reflectance spectra of the crystals were recorded using a home-built spectrometer on loan from Mobile Labs (CHARISMAA initiative) with a resolution of $8 \mathrm{~nm}$. NIR and MIR spectra of the crystalline adducts were recorded using, respectively, a Perkin Elmer Spectra One NIR spectrometer (4000-10000 $\mathrm{cm}^{-1}$ spectral range, $4 \mathrm{~cm}^{-1}$ resolution, 30 scans) and a Perkin Elmer Spectrum MIR spectrometer (600$3200 \mathrm{~cm}^{-1}$ range, $4 \mathrm{~cm}^{-1}$ resolution, 30 scans).

\section{Thermal analysis}

Differential-scanning caloriemetry (DSC) and thermogravimetric analysis (TGA) were carried out using a Rheometric instrument in simultaneous thermal-analysis mode with a $10{ }^{\circ} \mathrm{C} \min ^{-1}$ heating rate.

\section{Crystallography}

Single-crystal diffraction data for 1a-1c was obtained using an Oxford Xcalibur diffractometer with a Mo (K $\alpha)$ source (wavelength $0.71073 \AA$ ) at $180 \mathrm{~K}$. The structures were solved using SHELXL-97. ${ }^{41}$ The diffraction pattern of 1d was collected on a similar instrument at $150 \mathrm{~K}$, solved using SHELXT, ${ }^{42}$ and refined using the OSCAIL software. ${ }^{43}$ A comparison between powder patterns obtained from the filtrate and powder patterns simulated from the crystal structures showed a good match, suggesting that the products obtained from solution crystallisation do not contain residual starting materials (see Fig. S1-S4†).

\section{Computational modelling}

Computational modelling was carried out within the KohnSham density-functional theory framework, ${ }^{44,45}$ as implemented in the Vienna $A b$ Initio Simulation Package (VASP) code. ${ }^{46}$

Initial models of the crystal structures of 1a-1d were built from the X-ray structures. Additional gas-phase models of Lawsone, coformers a-d, the $\mathbf{d}_{2}$ dimer and the $\mathrm{H}$-bonded species 1a-1d were created by extracting the atomic coordinates of each species from the crystal structures and placing them in a simulation cell with an initial $10 \AA$ vacuum gap between the closest atoms in adjacent periodic images.

Geometry optimisations and initial total-energy calculations were carried out with the PBEsol functional ${ }^{47}$ with projector augmented-wave (PAW) pseudopotentials ${ }^{48,49}$ and a plane-wave kinetic-energy cutoff of $850 \mathrm{eV}$. In the calculations on the crystalline models, the Brillouin zone was sampled with $\Gamma$-centred Monkhorst-Pack $k$-point meshes ${ }^{50}$ with $1 \times 3 \times$ $1,3 \times 1 \times 1,1 \times 1 \times 3$ and $1 \times 3 \times 1$ subdivisions for $1 \mathrm{a}, \mathbf{1 b}, \mathbf{1 c}$ and 1d, respectively. This corresponds to two irreducible $k$-points in each structure. In the gas-phase calculations, the electronic wavefunctions were evaluated at the $\Gamma$ point. These convergence parameters were sufficient to converge the absolute total energies to within $1 \mathrm{meV}$ atom $^{-1}$, and the pressure to well within $1 \mathrm{kbar}(0.1 \mathrm{GPa})$. The electronic wavefunctions were optimised to a tolerance of $10^{-6} \mathrm{eV}$, and the positions of 
the ions, and also the cell shape/volume in the case of the crystalline models, were optimised until the magnitude of the forces on the ions was less than $10^{-2} \mathrm{eV} \AA^{-1}$.

Electronic-structure calculations, including the evaluation of energy gaps, the orbital analyses, and the computation of absorption spectra, were performed using the PBE0 hybrid functional $^{51}$ using the PBEsol-optimised structures as input. The absorption spectra were computed using the linearoptics routines in VASP, ${ }^{52}$ and the number of electronic bands was increased to $3 \times$ the default value in these calculations in order to ensure the convergence of the sum over empty states.

We note that, due to the size of the unit cell of the 1c crystal (536 atoms), we had to reduce the plane-wave cutoff to $550 \mathrm{eV}$ for the PBE0 calculations on this system. This is still above $1.3 \times$ the default cutoff recommended for the pseudopotentials we used, and so we expect it should give reasonable absorption spectra and charge/orbital densities; however, the total energies cannot be compared with those calculated using higher cutoffs, and so we did not include PBE0 formation energies for this compound in Table 1 (see discussion below).

\section{Results and discussion}

Fig. 1 shows the supramolecular organisation of the component molecules in $\mathbf{1 a - 1 d}$. The crystal-structure parameters from the single-crystal X-ray diffraction are reported in Table $\mathrm{S} 1, \dagger$ and the structures are available from the CCDC under the codes 1418634-1418637. While 1a, 1b and 1d crystallised in the monoclinic space group $P 2_{1} / n, 1 \mathrm{c}$ adopted the orthorhombic space group Fdd2. In 1a-1c, the coformers act as

Table 1 Calculated formation energies $\left(E_{\mathrm{F}}\right)$ of the gas-phase $\mathrm{H}$-bonded species and corresponding crystals of $1 \mathrm{a}-1 \mathrm{~d}$, plus the gas-phase homodimer of $\mathbf{d}\left(\mathbf{d}_{2}\right)$. Two sets of energies are given: the first were obtained from models fully relaxed with the PBEsol functional, while the second were obtained from single-point calculations on these models with the PBEO hybrid functional (denoted "PBE0@PBEsol"). In each set, the lefthand column gives the formation energies, while the right-hand one gives the (per $\mathrm{H}$-bonded unit) differences between the crystals and gasphase species. We note that PBEOCPBEsol values for the crystal of $1 \mathrm{c}$ are missing, as the size of the unit cell made it impractical to perform these calculations at the same level of accuracy as used in the calculations on the others

\begin{tabular}{llllll}
\hline & \multicolumn{2}{l}{$\left[\mathrm{kJ} \mathrm{mol}^{-1}\right.$ Adduct $\left.^{-1}\right]$} & & \\
\cline { 2 - 3 } Species & \multicolumn{2}{l}{ PBEsol } & & & \multicolumn{2}{l}{ PBE0@PBEsol } \\
\cline { 2 - 3 } \cline { 5 - 6 } & $E_{\mathrm{F}}$ & $\Delta E$ & & $E_{\mathrm{F}}$ & $\Delta E$ \\
\hline d homodimer & -104.17 & - & & -78.39 & - \\
Gas phase 1a & -87.19 & - & & -46.74 & - \\
Gas phase 1b & -89.25 & - & & -47.61 & - \\
Gas phase 1c & -92.28 & - & -50.71 & - \\
Gas phase 1d & -187.18 & - & & -130.16 & - \\
Crystal 1a & -161.35 & -74.15 & -96.87 & -50.13 \\
Crystal 1b & -185.46 & -96.20 & -110.47 & -62.87 \\
Crystal 1c & -176.64 & -84.36 & - & - \\
Crystal 1d & -234.21 & -47.03 & -154.16 & -24.00
\end{tabular}

(a) $1 \mathrm{a}$

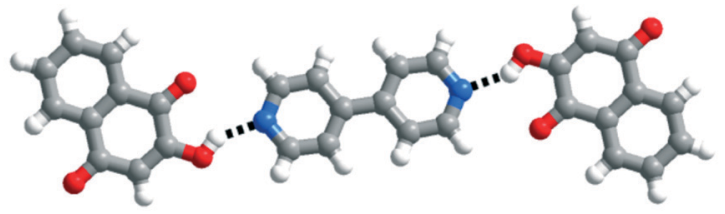

(b) $1 \mathbf{b}$

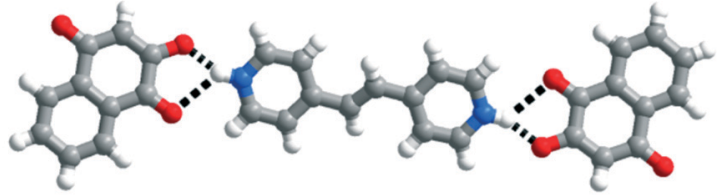

(c) $1 \mathrm{c}$

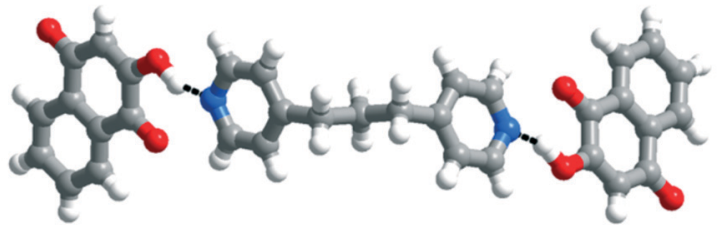

(d) $1 \mathrm{~d}$

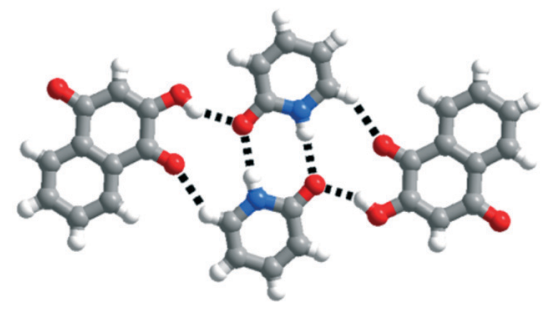

Fig. 1 Crystal packing in 1a (a), 1b (b), 1c (c) and 1d (d).

spacers, joining two molecules of $\mathbf{1}$ and forming discrete 3-membered supramolecular assemblies (Fig. 1a-c).

The Lawsone molecules and coformer are almost coplanar in 1a and $1 \mathrm{~b}$, whereas the Lawsone molecules are significantly tilted with respect to each other in 1c. In 1d, 2-hydroxy pyridine exists in its tautomeric form (2-pyridone), and two molecules of 2-pyridone interact to form dimers, which then act as spacers joining two molecules of 1 as in 1a-1c (Fig. 1d). In 1d, a dimer of $\mathbf{d}\left(\mathbf{d}_{2}\right)$ thus plays an equivalent role to coformers a-c in adducts $1 \mathbf{a}-\mathbf{1 c}$.

This gives rise to different Lawsone:coformer ratios in the crystalline adducts, viz. 2:1 and 1:1 stoichiometries in 1a-1c and $1 \mathrm{~d}$, respectively.

In 1a, 1c and 1d, 1 interacts with the coformers through neutral $\mathrm{O}-\mathrm{H} \cdots \mathrm{N}$ hydrogen bonds, whereas in $\mathbf{1 b}$ cations of $\mathbf{b}$ interact with anions of $\mathbf{1}$ by two $\mathrm{N}-\mathrm{H}^{+} \cdots \mathrm{O}^{-}$and $\mathrm{N}-\mathrm{H}^{+} \cdots \mathrm{O}$ hydrogen bonds. This is supported by NIR spectra (Fig. S5 $\dagger$ ), in which $\mathrm{O}-\mathrm{H}$ combination bands are seen in $1 \mathrm{a}$ and $1 \mathrm{c}$, but not in $\mathbf{1 b}$ (we also recorded standard IR spectra, which may be found in Fig. S6 $\dagger$ ). In general, proton transfer in cocrystallisation reactions is typically considered to be a function of $\Delta \mathrm{p} K_{\mathrm{a}} \cdot{ }^{53-55}$ As a point of terminology, the proton transfer exhibited by $\mathbf{1 b}$ in the solid state means this is a salt, whereas a cocrystal is typically taken to be formed through (formally) neutral intermolecular interactions. We therefore refer to the solid forms of $\mathbf{1 a - 1 d}$ as "crystalline adducts" in the following discussion.

In 1d, the NIR combination bands observed are of amide III groups. ${ }^{56}$ This system is peculiar, in that $\mathbf{d}$ undergoes tautomerisation to give 2-pyridone, as observed elsewhere in 
the literature. ${ }^{57}$ The formation of a zwitterion can be ruled out, since the $\mathrm{C}-\mathrm{O}$ bond distance of $1.26 \AA$ is suggestive of a double bond, and the melting point determined by DSC was much lower than that of the salt $\mathbf{1 b}$ (see Fig. S7 $\dagger$ ). A similar bond length has been observed in other literature studies. ${ }^{58}$ A six-membered ring is formed through intermolecular hydrogen bonding between the two 2-pyridone molecules, and the carbonyl oxygen of 2-pyridone also forms intermolecular hydrogen bonds with the hydroxyl group of Lawsone. Such homodimer formation has also been observed in cocrystals of carbamazipine, where the homosynthon formed by the amide hydrogen bonding forms $1 \mathrm{D} \mathrm{H}$-bonds with the coformer. ${ }^{59}$ From here on, $\mathbf{d}$, the $\mathbf{d}_{2}$ dimer and $1 \mathbf{d}$ will refer to 2-pyridone, its dimer, and its adduct with Lawsone, respectively, where appropriate, rather than to species derived from the initial 2-hydroxy pyridine.

Coformers $\mathbf{b}$ and $\mathbf{d}$ are both known to undergo photodimerisation reactions. ${ }^{58,60}$ However, in $\mathbf{1 b}$ the distances between the $\mathrm{C}=\mathrm{C}$ bonds in $\mathrm{b}$ in adjacent layers is $3.816 \AA$, while the distance between molecules of $\mathbf{d}$ in $1 \mathrm{~d}$ is $5.153 \AA$. These distances are both longer than the ideal $\mathrm{C}=\mathrm{C}$ distance for photodimerisation $(1.727(4)-3.324(4) \AA$ ), and hence we do not expect to see this phenomenon in our systems. ${ }^{58}$

To study the crystal morphologies, we recorded brightfield microscope images of the four crystalline adducts (Fig. 2). 1a formed block crystals, while 1b formed bladed crystals and 1c and 1d both formed needle-like crystals. We further characterised 1a-1d by differential-scanning calorimetry (DSC; see Fig. S7†). The DSC traces show $\mathbf{1 b}$ to have the highest melting point of $210.10{ }^{\circ} \mathrm{C}$, compared to 183.96 and $155.80{ }^{\circ} \mathrm{C}$ for $1 \mathrm{a}$ and 1c, respectively. This is naturally accounted for by the stronger ionic hydrogen bonds in $\mathbf{1 b}$. The melting point of $1 \mathrm{~d}$ was recorded to be $147.90{ }^{\circ} \mathrm{C}$.

Adduct formation also led to clear differences in colour with respect to neat Lawsone (Fig. 3). While the crystalline adducts of 1 with a and d displayed hypsochromic shifts in

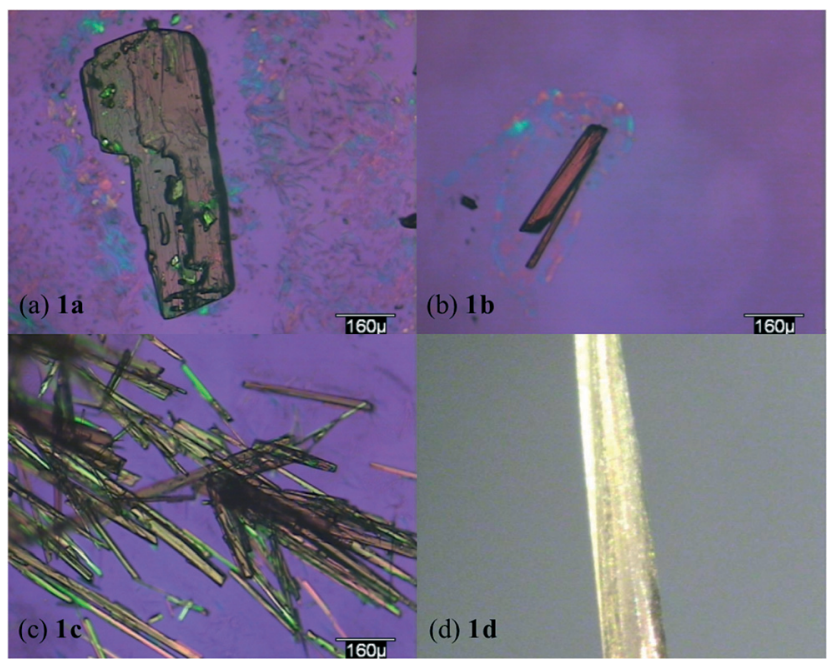

Fig. 2 Bright-field microscope images of the crystals of 1a-1c, taken under polarised light $(\mathrm{a}-\mathrm{c})$, together with an image of a single crystal of $1 d$ mounted on a diffractometer pin (d).

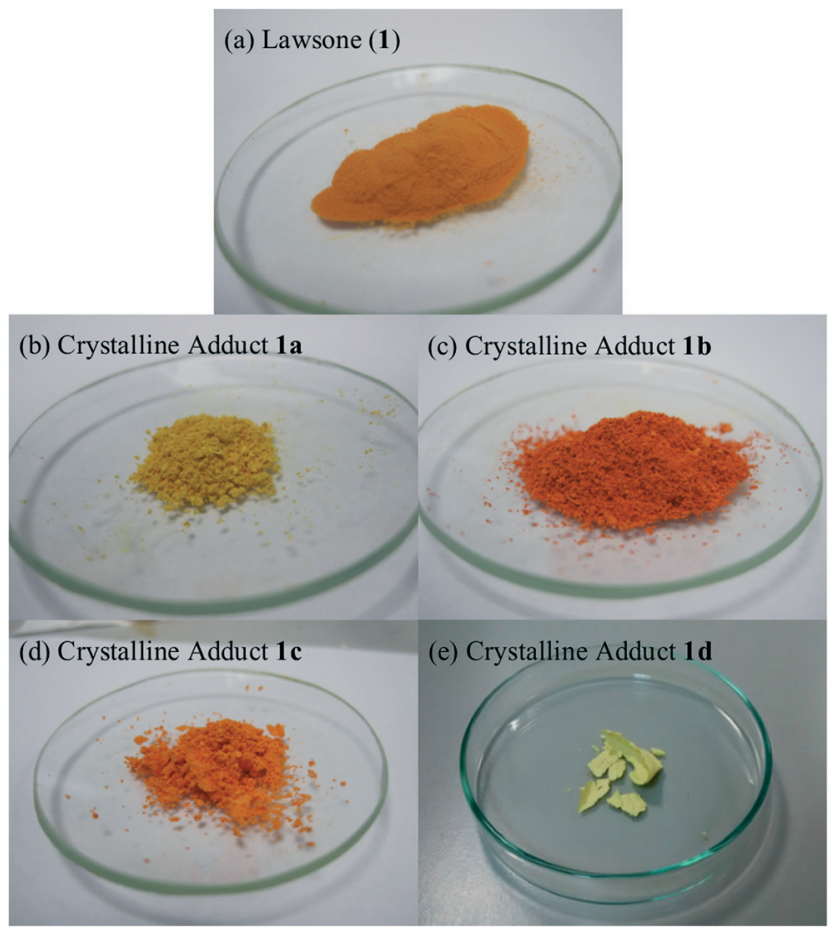

Fig. 3 Photographs of Lawsone $(1 ;$ a) and the crystalline adducts synthesised using 4,4'-bipyridine (1a; b), 4-(2-pyridine-4-ethyl)pyridine (1b; c), 1,3-di(4-pyridyl)propane (1c; d) and 2-hydroxy pyridine (1d, e), illustrating the observed colour changes.

colour, bathochromic colour shifts were obsered in $\mathbf{1 b}$ and 1c. Differences in the colour of neat Lawsone and $\mathbf{1 a - 1 d}$ are clearly evident in the UV-visible reflectance spectra of the crystals (Fig. 4a). We also recorded solution spectra (Fig. 4b) in a 2:1 mixture of acetonitrile and methanol, to retain the H-bonded species. These spectra show small shoulder-like features in the visible region in the case of $\mathbf{1 b}$ and $\mathbf{1 c}$, but these were weak in comparison to the absorption in the UV. These features were not as prominent in the spectra of 1a and $1 \mathrm{~d}$ as in the spectra of the other two adducts.

To better understand the energetics of the adduct formation and the origin of the colour changes, we carried out complementary theoretical modelling within the densityfunctional theory (DFT) formalism.

As a starting point, we first fully relaxed the crystal structures of 1a-1d with the PBEsol functional, ${ }^{47}$ optimising both the positions of the atoms and the unit-cell shapes/volumes. We also relaxed gas-phase models of Lawsone and the four coformers a-d, the $\mathbf{d}_{2}$ homodimer, and the H-bonded species 1a-1d, all of which were made by extracting the relevant species from the collected crystal structures. Finally, to obtain more accurate energetics and electronic structures, and to calculate the optical-absorption spectra of the solid-state species, we performed single-point calculations on the PBEsoloptimised models using the PBE0 hybrid functional. ${ }^{51}$

The calculated unit-cell parameters are compared to the experimental structures in Table S2. $\uparrow$ We found that PBEsol seemed to give a fairly good reproduction of the structures, 

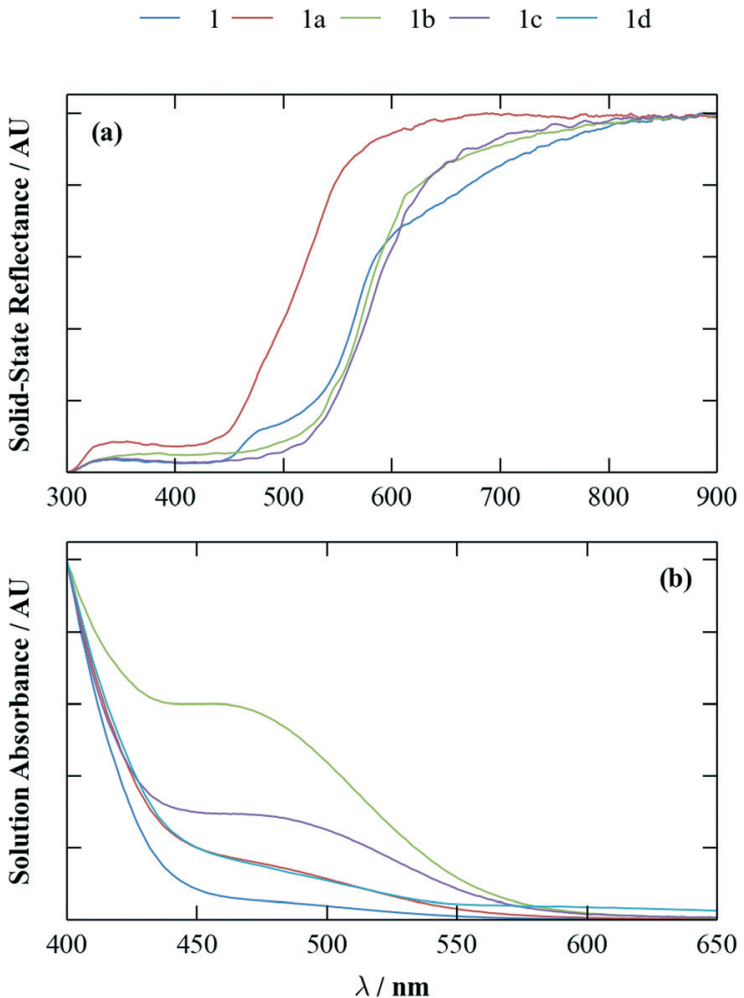

Fig. 4 Optical properties of Lawsone (1) and the crystalline adducts 1a-1d. The two plots show the normalised solid-state reflectance spectra of 1 and $1 \mathrm{a}-1 \mathrm{c}(\mathrm{a})$, and the solution absorbance spectra of 1 and 1a-1d (b), recorded between 300-900 and 400-650 nm, respectively. The solution spectra were recorded in a $2: 1$ mixture of acetonitrile and methanol in order to retain the $\mathrm{H}$-bonded species. To better identify the position of the transition edges in the reflectance spectra, the first derivatives were also calculated by numerical differentiation (see Fig. S8†).

with a tendency to overestimate the cell volume but with $<5 \%$ variation in most parameters. Without measuring the thermal expansion of the crystals, to check how steeply the lattice parameters change with temperature, we would not expect a perfect match between the calculations and the experimental structures, since the DFT optimisation is "athermal", i.e. is performed at $0 \mathrm{~K}$ and in the absence of lattice-dynamical effects such as the vibrational zero-point energy, while the crystal structures were collected at finite temperature. However, the discrepancy in the cell volume in 1a-1c was found to be mostly due to a consistent overestimate of the length of the short lattice vectors, which is most likely an indicator that PBEsol is not able to describe fully the attractive part of the dispersive interaction between molecules along the stacking direction. In support of this, the single-point PBE0 calculations predicted negative cell pressures, implying that a more accurate functional would indeed lead to a reduction in the cell volume, although we note that PBE0 would also not necessarily describe the dispersion interactions correctly. While internal relaxation of the atomic coordinates occurred during the optimisation of 1d, the calculations predicted no change to the cell shape or volume.
After optimising the $\mathrm{H}$-bonded species in the gas phase, we found they generally retained a structure similar to that in the solid form, with the exception of the proton transfer in 1b. In both the experimental and the PBEsol-optimised crystal structures, the proton appears to be shared equally between the coformer $\mathrm{N}$ and the Lawsone $\mathrm{O}$. After optimisation of the gas-phase adduct, however, it appears to be mainly associated with the latter, as is the case in the other crystalline adducts. Images of the optimised gas-phase adduct and crystal structure of $\mathbf{1 b}$ are given in Fig. $\mathrm{S} 9 \dagger$ for comparison. This observation suggests that the proton transfer is a consequence of the crystal packing, and the resultant intermolecular interactions, in the solid form.

To investigate the energetics of the adduct formation, we calculated the formation energies $\left(E_{\mathrm{F}}\right)$ of the gas-phase H-bonded species and crystals of 1a-1d (Table 1). To a first approximation, the formation energies of the gas-phase species correspond to the energies of the H-bonding interactions, while the (per-unit) differences in $E_{\mathrm{F}}$ between the gasphase species and crystals gives an indication of the strength of the interactions between the constituent components in the solid state.

For 1a-1c, the PBEsol and PBE0 calculations predict the energy of each Lawsone-coformer $\mathrm{H}$ bond to be on the order of 45 and $25 \mathrm{~kJ} \mathrm{~mol}^{-1}$, respectively, increasing in the order $\mathbf{c}$ $>\mathbf{b}>\mathbf{a}$. In adduct $1 \mathrm{~d}$, there are two types of $\mathrm{H}$ bond, viz. those between the two 2-pyridone molecules, and those between the $\mathbf{d}_{2}$ dimer and Lawsone; for the present discussion, we estimate the two contributions from the difference in the formation energies of the $\mathbf{d}_{2}$ dimer and the gas-phase 1d adduct. The d-d interaction is stronger than the bond between the dimer and Lawsone at $\sim 50 / 40$, and $40 / 25 \mathrm{~kJ}$ $\mathrm{mol}^{-1}$ per bond with PBEsol and PBE0, respectively. Interestingly, PBEsol predicts the bond between the $\mathbf{d}_{2}$ dimer and Lawsone to be the weaker of the four, whereas PBE0 predicts it to be the strongest. Nonetheless, the range in the calculated bond strengths between Lawsone and $\mathbf{a}-\mathbf{c} / \mathbf{d}_{2}$ is fairly small at $<5$ (PBEsol) and $<2.5$ (PBE0) $\mathrm{kJ} \mathrm{mol}^{-1}$.

For 1a-1c, the per-unit energy gain due to the intermolecular interactions in the crystal is of a similar magnitude to the $\mathrm{H}$-bonding in the gas phase, being around 85 and $55 \mathrm{~kJ} \mathrm{~mol}^{-1}$ with PBEsol and PBE0, respectively, for 1a-1c. 1d behaves a little differently in this respect, with the energy difference between the gas phase and solid forms being around half that between the gas-phase adduct and the isolated Lawsone and $\mathbf{d}_{2}$ species. The reasons for this are not clear, but this analysis nonetheless provides some interesting insight into the relative energetic stabilisation provided by $\mathrm{H}$-bonding and dispersive interactions.

Next, we used the PBE0 calculations to investigate the electronic structures of the molecules, gas-phase H-bonded species and crystals. Fig. 5 shows the simulated absorption spectra of crystals 1a-1d. The four spectra are qualitatively similar in form, with a handful of features between 300-400 $\mathrm{nm}$, a steep rise below approx. $250 \mathrm{~nm}$, and a smooth tail off above $400 \mathrm{~nm}$. The spectra in Fig. 4(b) were recorded only to 


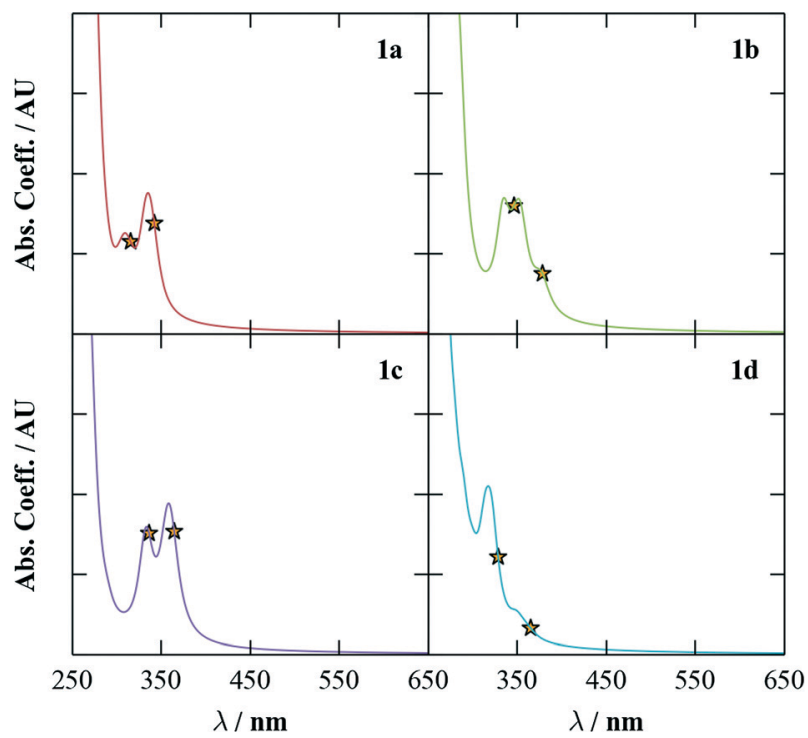

Fig. 5 Simulated absorption spectra of crystals $1 \mathrm{a}-1 \mathrm{~d}$ in the range 250-650 nm, obtained at the PBEO hybrid level of theory on the PBEsol-relaxed structures. The yellow stars mark the positions of the direct band gaps at the two irreducible $k$-points modelled in each structure.

$400 \mathrm{~nm}$, so it is difficult to assess the correspondence between these and the calculated absorption profiles. However, most appear to display the same long tails, and the small shoulder at $\sim 375 \mathrm{~nm}$ in the calculated spectrum of $\mathbf{1 b}$ might be equated to the broad absorption at $\sim 475 \mathrm{~nm}$ in the corresponding spectrum in Fig. 4. If this is the case, it implies that the calculated spectra are blue shifted by around $100 \mathrm{~nm}$ with respect to the experimentally-recorded ones, which can be attributed to the approximations in the timedependent DFT (TD-DFT) method employed in these simulations. ${ }^{61}$

A feature highlighted in Fig. 5 is that in all four crystals the main absorption features match up quite well with the energy gap between the highest-occupied and lowestunoccupied crystal orbitals (HOCOs/LUCOs) at the two irreducible $k$-points used to model the electronic structure. This implies that an analysis of the frontier orbitals and energy gaps in the crystals and component species may provide some insight into the origin of the colour changes observed following adduct formation. Table 2 compares the calculated gaps in Lawsone (1), coformers $\mathbf{a}-\mathbf{d}$, the $\mathbf{d}_{2}$ dimer, and $\mathbf{1 a - 1 d}$ in their gas-phase and crystalline forms.

Lawsone has a smaller energy gap than any of the four coformers, being $>1 \mathrm{eV}$ narrower than the gaps of coformers a and $\mathbf{c}$, approx. $1 \mathrm{eV}$ narrower than the energy gaps of $\mathbf{d}$ and its dimer, which are fairly similar, and around $400 \mathrm{meV}$ narrower than the HOMO-LUMO gap of $\mathbf{b}$. The gaps of $\mathbf{a}, \mathbf{b}$ and $\mathbf{c}$ fall into the order $\mathbf{c}>\mathbf{a}>\mathbf{b}$, which is consistent with the degree of conjugation in these molecules. Taking the gasphase energy gap of Lawsone as a reference, the gaps of the $\mathrm{H}$-bonded species are consistently smaller by $200-400 \mathrm{meV}$. In all four of the solid-state structures, convergence testing
Table 2 Calculated energy gaps $\left(E_{\mathrm{g}}\right)$ of Lawsone (1), coformers a- $d$ and the homodimer of $\mathbf{d}\left(\mathbf{d}_{2}\right)$, the gas-phase $\mathrm{H}$-bonded species $1 \mathrm{a}-1 \mathrm{~d}$, and the corresponding crystal structures. For the latter, two gaps are given, one for each of the $k$-points used to model the wavefunctions. For the gasphase species and crystals, the differences in energy gap compared to the gas-phase Lawsone molecule are shown in the adjacent columns

\begin{tabular}{lllll}
\hline Species & $E_{\mathrm{g}, 1} \mathrm{eV}^{-1}(\mathrm{~nm})$ & $\Delta 1 \mathrm{meV}^{-1}$ & $E_{\mathrm{g}, 2} \mathrm{eV}^{-1}(\mathrm{~nm})$ & $\Delta 1 \mathrm{meV}^{-1}$ \\
\hline Lawsone (1) & $4.121(301)$ & - & - & - \\
Coformer a & $5.544(224)$ & - & - & - \\
Coformer b & $4.508(275)$ & - & - & - \\
Coformer c & $6.357(195)$ & - & - & - \\
Coformer d & $5.105(243)$ & - & - & - \\
d homodimer & $5.141(241)$ & - & - & - \\
Gas-phase 1a & $3.907(317)$ & -214 & - & - \\
Gas-phase 1b & $3.767(329)$ & -355 & - & - \\
Gas-phase 1c & $3.887(319)$ & -234 & - & - \\
Gas-phase 1d & $3.732(332)$ & -389 & - & - \\
Crystal 1a & $3.927(316)$ & -195 & $3.622(342)$ & -500 \\
Crystal 1b & $3.579(346)$ & -543 & $3.277(378)$ & -844 \\
Crystal 1c & $3.685(336)$ & -436 & $3.400(365)$ & -722 \\
Crystal 1d & $3.774(329)$ & -348 & $3.396(365)$ & -726 \\
\end{tabular}

found that two $k$-points were required along the short lattice vector, corresponding to the stacking direction, to describe the electronic wavefunctions, which implies that there are significant interactions between the localised orbitals of adjacent units in the electronic bands of the crystal. The HOCOLUCO gaps at these two $\boldsymbol{k}$-points are generally narrowed by 200-500 meV with respect to those of the corresponding gasphase species, with the exception being the gaps of $1 \mathrm{a}$ and 1d at the zone centre $(\Gamma)$, which show a relatively small widening of 20 and $40 \mathrm{meV}$, respectively.

By analysing orbital-density plots obtained from the PBE0 calculations, we found that the forms of the HOMOs and LUMOs of the $\mathrm{H}$-bonded species could be qualitatively well understood in terms of the frontier orbitals of the component molecules. In 1a and 1c, the HOMO and LUMO are both linear combinations of the corresponding Lawsone orbitals. In $\mathbf{1 b}$, the HOMO is on the Lawsone molecules, whereas the LUMO resides on the coformer, and the reverse occurs in $\mathbf{1 d}$. There is little evidence of electronic delocalisation between the Lawsone and coformer molecules in the H-bonded species, nor, by extension, between the Lawsone molecules across the bridging coformers. In all four systems, the HOMO and LUMOs of the gas-phase species match up very well with the HOCOs and LUCOs of the crystals, respectively, with the orbital densities at both $k$-points being visually near identical. An example orbital-density analysis for 1a is illustrated in Fig. 6, and similar analyses for 1b-1d may be found in Fig. S10-S16. $\dagger$

In 1a and 1c, the HOMO and LUMO in the adducts are both on Lawsone, so it is reasonable to suggest that the narrowing of the gap is due to the frontier orbitals on Lawsone being perturbed by the $\mathrm{H}$-bonding interaction with the coformer. In $\mathbf{1 b}$ and $\mathbf{1 d}$, the coformer provides a lower-energy LUMO and higher-energy HOMO, respectively, and so the narrowing of the gap with respect to neat Lawsone cannot be attributed solely to the $\mathrm{H}$-bonding interaction in this way. It is worth noting, however, that this may account for why the 

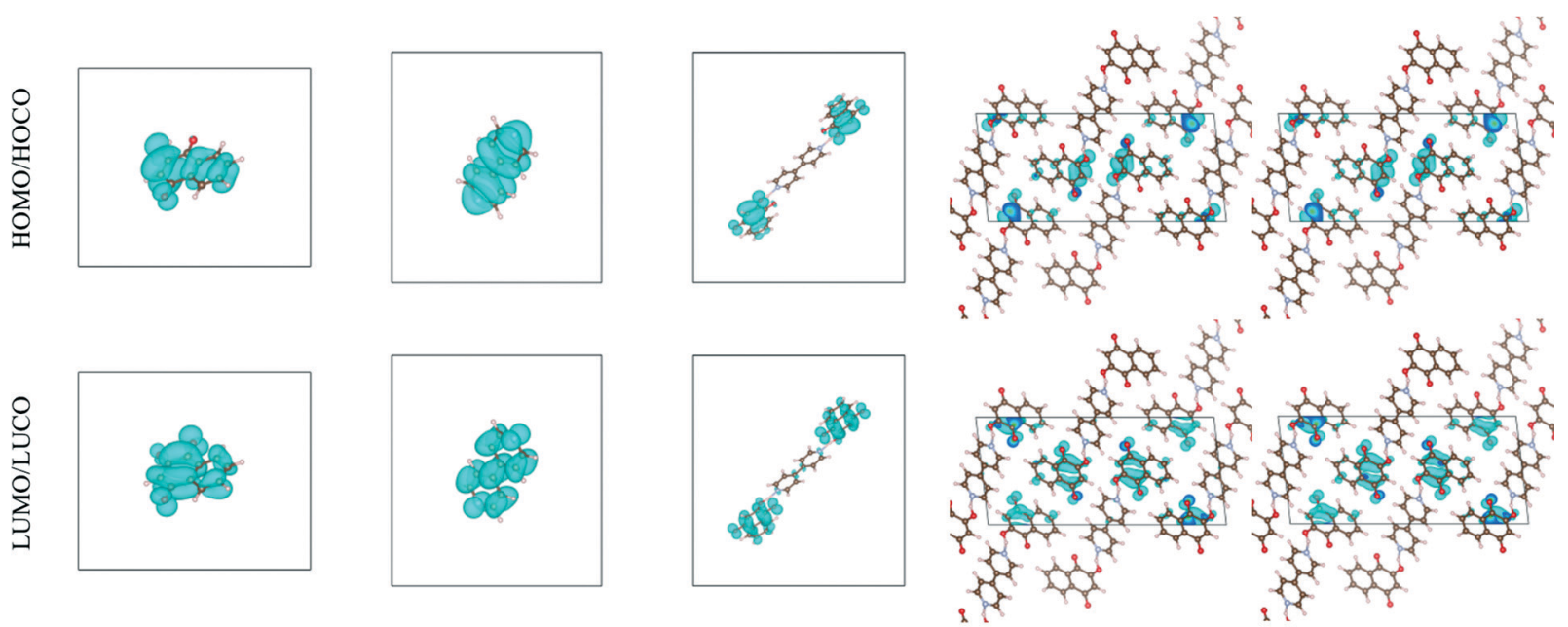

$\boldsymbol{k}$-point $1(\Gamma)$

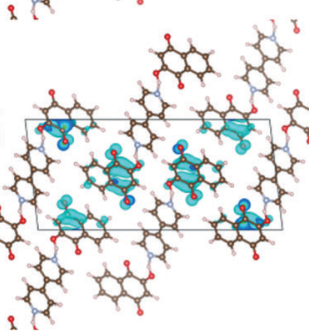

$k$-point 2

Lawsone (1) $\quad+$ Coformer $\mathbf{a} \quad=\quad$ Adduct $\mathbf{1} \mathbf{a} \quad>$

Crystal 1a

Fig. 6 Orbital-density plots illustrating the relationship between the frontier orbitals of Lawsone (1), coformer a, the gas-phase H-bonded 1a, and the corresponding solid form. For the latter, the frontier orbitals at both irreducible $k$-points used to model the electronic structure are shown (see discussion in text).

gap of these species undergoes a larger shift with respect to Lawsone than those of 1a and 1c.

To explore this further, and to investigate how reliably the frontier orbitals of the isolated component molecules might be used to predict those of the H-bonded species (and hence the crystals), we constructed orbital-alignment diagrams matching up the HOMOs and LUMOs of Lawsone and the coformers with the four highest-occupied and lowestunoccupied orbitals of the corresponding gas-phase adducts (Fig. 7). The reason for our considering four orbitals is that the molecular orbitals from the two Lawsone molecules in the $\mathrm{H}$-bonded species generally formed (near-) degenerate pairs, presumably corresponding to in-phase and antiphase combinations. When performing the alignment, the orbital energies were adjusted to the electrostatic potential in the vacuum region of the cells. ${ }^{62}$ We note that this procedure cannot be applied to bulk materials (at least not those without a sufficiently large internal pore ${ }^{62}$ ), and so we were unable to include the crystals in our alignment.

The analysis in Fig. 7 shows that, in general, the frontier orbitals of Lawsone and the coformer are significantly perturbed on forming the $\mathrm{H}$-bonded complexes. The $\mathrm{H}$ bonding appears to cause the Lawsone orbitals to shift to higher energies, while those of the coformer are lowered with respect to the isolated molecule.

In the case of 1a and 1c, for which the HOMO and LUMO are both Lawsone-based, the HOMO of the coformer is below that of Lawsone, and thus the stabilisation due to the $\mathrm{H}$ bonding serves to push it further below the Lawsone orbitals. Although the coformer LUMO is lowered in energy, this is insufficient to bring it below the Lawsone-based LUMO observed in these species, although the alignment diagram for 1a shows that they are close in energy in this system. In both species, the primary origin of the colour shift in the gas phase can thus be ascribed to a slight difference in the relative destabilisation of the Lawsone HOMO and LUMO, which serves overall to narrow the energy gap.

Considering the other two species, in $\mathbf{1 b}$ the destabilisation of the Lawsone-based LUMO and stabilisation
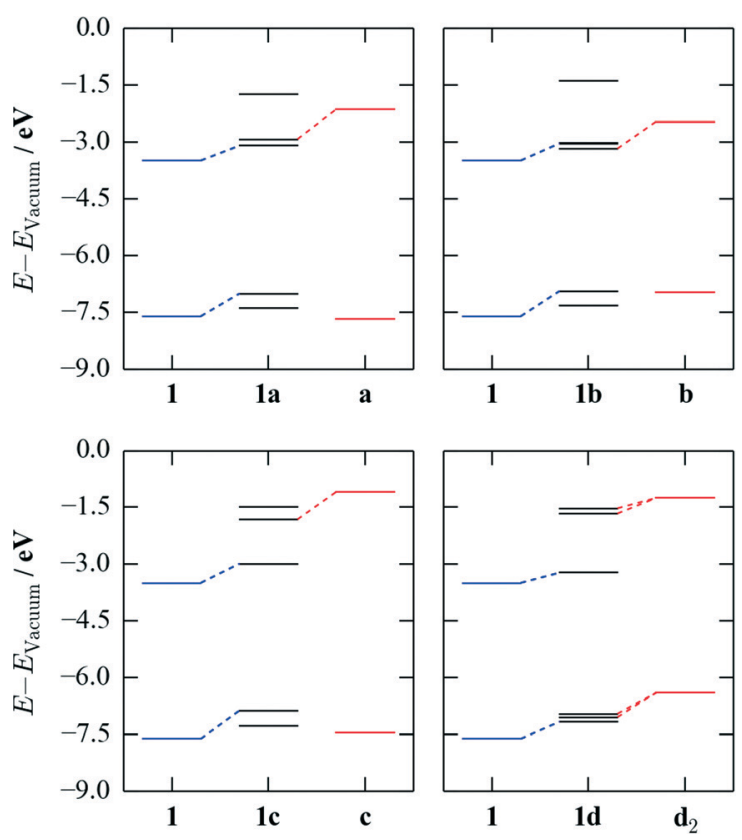

Fig. 7 Orbital-alignment diagrams for the gas-phase $\mathrm{H}$-bonded species 1a-1d. Each plot shows the position of the HOMO and LUMO of Lawsone (blue) and the coformer (red) with respect to the four highestoccupied and lowest-unoccupied orbitals of the adduct (black). Dashed lines are drawn between orbitals in the component species which are related to those in the adduct. All energies are expressed relative to the vacuum level. For adduct $1 \mathrm{~d}$, the $\mathrm{d}_{2}$ dimer, rather than the isolated molecule, was used as the coformer in the alignment. 
of the coformer orbitals is sufficient to push the LUMO onto the coformer. In 1d, the HOMO on the coformer is above that of Lawsone, and in this case the rearrangement of the orbital energies is insufficient to swap the order, leading to a coformer-based HOMO in this species, although as for the LUMOs in 1a the two HOMOs are close in energy.

The energy shifts make it somewhat difficult to predict $a$ priori the relationship between the frontier orbitals in Lawsone and the coformers and those in the corresponding $\mathrm{H}$-bonded species. However, given that the direction of the shift appears to be a consistent trend, we can extract two general observations: (1) if the HOMO on the coformer is below or similar in energy to that on Lawsone, the Lawsone orbitals will likely become the HOMO in the adduct, and (2) if the LUMOs on the two components are similar in energy, or if the orbital on the coformer is below the Lawsone LUMO, the LUMO of the adduct will likely be coformer based. Using these principles, it may be possible to assess qualitatively how other potential coformers may shift the colour of neat Lawsone using relatively cheap gas-phase calculations.

\section{Conclusions}

Four crystalline adducts of the Lawsone molecule with bipyridine, ethylenebipyridine, propylenebipyridine and 2-hydroxy pyridine were synthesised and characterised using single-crystal X-ray diffraction, thermal analysis, and UV/visible and IR spectroscopy. Three of the coformers yielded crystals based around three-membered complexes consisting of one conformer and two Lawsone molecules, while the fourth gave a 1:1 complex with a similar structure based around a $\mathrm{H}$-bonded dimer of the coformer. Whereas Lawsone and the coformer in the bipyridine (1a) and propylenebipyridine (1c) adducts interact through neutral $\mathrm{H}$ bonds, the ethylenebipyridine (1b) system shows a solid-state proton transfer, which is also clearly evident from its IR spectrum. 2-hydroxy pyridine undergoes tautomerisation and forms homodimers that then hydrogen bond with the Lawsone molecules. The crystalline adducts were all found to exhibit visible shifts in colour with respect to neat Lawsone.

The hierarchy of interactions in the adduct formation, viz. $\mathrm{H}$-bonding between Lawsone and the coformers and dispersive interactions in the solid state, allowed us to use computational modelling to study the contributions of the various effects to the energetics and colour shift. Both interactions lead to roughly equal energetic gains on adduct formation, and the typical strength of $\mathrm{H}$-bonding interactions between Lawsone and one of the coformers was calculated to be on the order of $25 \mathrm{~kJ} \mathrm{~mol}^{-1}$ from single-point PBE0 calculations on PBEsol-optimised structures. It was also noted that the proton transfer in $\mathbf{1 b}$ was not observed in the gas-phase adduct, suggesting that this is a product of the intermolecular interactions in the solid state. The longwavelength absorption features in the spectra of the crystalline adducts were found to be relatable to the size of the HOCO-LUCO gap, which could be rationalised in terms of the frontier orbitals of Lawsone and the coformer. From our electronic-structure analyses, the H-bonding appears to raise and lower the energies of the Lawsone and coformer orbitals, respectively, ultimately leading to the energy gaps of the H-bonded gas-phase species being 200-400 meV lower than those of a gas-phase Lawsone molecule. The intermolecular interactions between units in the crystal lead to a further narrowing of the gap of a similar magnitude.

In summary, the combined experimental and theoretical approach taken in this study has allowed us to gain some interesting insight into the energetics of the adduct formation and and origin of the colour shift in crystalline adducts of the Lawsone molecule, which we hope will contribute to future crystal-engineering studies on this and related systems.

\section{Acknowledgements}

The authors are grateful to Dr John E. Davies for collecting single-crystal X-ray data for some of the reported crystalline adducts. AP is grateful to Prof. Stephen R. Elliott for hosting her during her doctoral studies. AP is also grateful to MOLABS, part of a European initiative called CHARISMAA, for lending their reflectance spectrometer. JMS gratefully acknowledges financial support from an EPSRC Programme Grant (no. EP/K004956/1), plus the support of a Trinity College Internal Graduate Studentship award during his doctoral studies. The computational modelling was carried out using the Balena HPC system, maintained by the Bath University Computing Service, and the ARCHER supercomputer, accessed through membership of the UK HPC Materials Chemistry Consortium, which is funded by EPSRC grant no. EP/L000202. AD thanks the Pfizer Institute for Pharmaceutical Materials Science for funding.

\section{Notes and references}

1 A. C. Dweck, Int. J. Cosmet. Sci., 2002, 24.

2 N. M. Rahmouna, Z. Boucherit-Otmania, K. Boucherita, M. Benabdallahb, D. Villeminc and N. Choukchou-Brahamb, Antibacterial and antifungal activity of lawsone and novel naphthoquinone derivatives, Médecine et Maladies Infectieuses, 2012, 42(6), 270-275.

3 N. Sritrairat, N. Nukul, P. Inthasame, A. Sansuk, J. Prasirt, T. Leewatthanakorn, U. Piamsawad, A. Dejrudee, P. Panichayupakaranant, K. Pangsomboon, N. Chanowanna, J. Hintao, R. Teanpaisan, W. Chaethong, P. Yongstar, N. Pruphetkaew, V. Chongsuvivatwong and W. Nittayananta, J. Oral Pathol. Med., 2011, 42(6), 270-275.

4 A. Ostovari, S. M. Hoseinieh, M. Peikari, S. R. Shadizadeh and S. J. Hashemi, Corros. Sci., 2009, 51, 1935-1949.

5 S. B. Zaware, R. G. Gonnade, D. Srinivas, A. Khan and S. Y. Rane, New J. Chem., 2011, 35.

6 Y. Yasin, N. M. Ismail, M. Z. Hussein and N. Aminudin, J. Biomed. Nanotechnol., 2011, 7.

7 R. Jelly, S. W. Lewis, C. Lennard, K. F. Lim and J. Almog, Chem. Commun., 2008, 3513-3515. 
8 A. V. Todkary, R. Dalvi, S. S. Alunke-Gawali, J. Linares, F. Varret, J. Marrot, J. V. Yakhmi, M. Bhadbhade, D. Srinivas, S. P. Gejji and S. Y. Rane, Spectrochim. Acta, Part A, 2006, 63, 130-138.

9 S. Salunke-Gawali, L. Kathawate, Y. Shinde, V. G. Puranik and T. Weyhermueller, J. Mol. Struct., 2012, 1010.

10 A. P. Neves, M. D. Vargas, C. A. Tellez Soto, J. M. Ramos, L. D. C. Visentin, C. B. Pinheiro, A. S. Mangrich and E. I. P. de Rezende, Spectrochim. Acta, Part A, 2012, 94.

11 C. E. Dalgliesh, J. Am. Chem. Soc., 1949, 71.

12 F. Zsila and I. Fitos, Org. Biomol. Chem., 2010, 8.

13 A. Delori, P. T. A. Galek, E. Pidcock and W. Jones, Chem. Eur. J., 2012, 18, 6835-6846.

14 A. Delori, P. Galek, E. Pidcock, M. Patni and W. Jones, CrystEngComm, 2013, 15, 2916-2928.

15 P. T. A. Galek, E. Pidcock, P. A. Wood, I. J. Bruno and C. R. Groom, CrystEngComm, 2012, 14, 2391-2403.

16 A. Delori, P. Maclure, R. M. Bhardwaj, A. Johnston, A. J. Florence, O. Sutcliffe and I. D. H. Oswald, CrystEngComm, 2014, 16, 5827-5831.

17 R. Thakuria, A. Delori, W. Jones, M. P. Lipert, L. Roy and N. Rodríguez-Hornedo, Int. J. Pharm., 2013, 453, 101-125.

18 N. J. Babu and A. Nangia, Cryst. Growth Des., 2011, 11, 2662-2679.

19 C. B. Aakeröy, S. Forbes and J. Desper, J. Am. Chem. Soc., 2009, 131, 17048-17049.

20 J. F. Remenar, S. L. Morissette, M. L. Peterson, B. Moulton, J. M. MacPhee, H. R. Guzmán and Ö. Almarsson, J. Am. Chem. Soc., 2003, 125, 8456-8457.

21 S. J. Bethune, N. Huang, A. Jayasankar and N. RodríguezHornedo, Cryst. Growth Des., 2009, 9, 3976-3988.

22 R. Banerjee, P. M. Bhatt, N. V. Ravindra and G. R. Desiraju, Cryst. Growth Des., 2005, 5, 2299-2309.

23 D. Yan, D. K. Bučar, A. Delori, B. Patel, G. O. Lloyd, W. Jones and X. Duan, Chem. Eur. J., 2013, 19, 8213-8219.

24 D. Yan, A. Delori, G. O. Lloyd, T. Friščić, G. M. Day, W. Jones, J. Lu, M. Wei, D. G. Evans and X. Duan, Angew. Chem., Int. Ed., 2011, 50, 12483-12486.

25 C. L. Jones, C. C. Wilson and L. H. Thomas, CrystEngComm, 2014, 16, 5849-5858.

26 D.-K. Bučar, S. Filip, M. Arhangelskis, G. O. Lloyd and W. Jones, CrystEngComm, 2013, 15, 6289-6291.

27 D. Yan, A. Delori, G. O. Lloyd, B. Patel, T. Friščić, G. M. Day, B. Dejan-Kresimir, W. Jones, J. Lu, M. Wei, D. G. Evans and X. Duan, CrystEngComm, 2012, 14, 5121-5123.

28 J. D. Wuest, Nat. Chem., 2012, 4, 74-75.

29 D. Yan, G. O. Lloyd, A. Delori, W. Jones and X. Duan, ChemPlusChem, 2012, 77, 1112-1118.

30 R. D. Bailey Walsh, M. W. Bradner, S. Fleischman, L. A. Morales, B. Moulton, N. Rodríguez-Hornedo and M. J. Zaworotko, Chem. Commun., 2003, 186-187.

31 S. Karki, T. Friščić, L. Fábián, P. R. Laity, G. M. Day and W. Jones, Adv. Mater., 2009, 21, 3905-3909.

32 S. R. Perumalla, L. Shi and C. C. Sun, CrystEngComm, 2012, 14, 2389-2390.

33 M. L. Cheney, N. Shan, E. R. Healey, M. Hanna, L. Wojtas, M. J. Zaworotko, V. Sava, S. J. Song and J. R. Sanchez-Ramos, Cryst. Growth Des., 2010, 10, 394-405.
34 P. Sanphui, N. R. Goud, U. B. R. Khandavilli, S. Bhanoth and A. Nangia, Chem. Commun., 2011, 47, 5013-5015.

35 S. Basavoju, D. Boström and S. P. Velaga, Pharm. Res., 2008, 25, 530-541.

36 A. V. Trask, W. D. S. Motherwell and W. Jones, Int. J. Pharm., 2006, 320, 114-123.

37 A. V. Trask, W. D. S. Motherwell and W. Jones, Cryst. Growth Des., 2005, 5, 1013-1021.

38 S. Ghosh and C. M. Reddy, Angew. Chem., Int. Ed., 2012, 51, 10319-10323.

39 C. L. Jones, C. C. Wilson and L. H. Thomas, CrystEngComm, 2014, 16, 5849-5858.

40 J. R. G. Sander, D.-K. Bučar, R. F. Henry, J. Baltrusaitis, G. G. Z. Zhang and L. R. MacGillivray, J. Pharm. Sci., 2010, 99, 3676-3683.

41 G. M. Sheldrick, Acta Crystallogr., Sect. C: Struct. Chem., 2015, 71, 3-8.

42 G. M. Sheldrick, Acta Crystallogr., Sect. A: Found. Adv., 2015, 71, 3-8.

43 P. McArdle, Y. Hu, A. Lyons and R. Dark, CrystEngComm, 2010, 12, 3119-3125.

44 W. Kohn and L. J. Sham, Phys. Rev., 1965, 140, 1133-1138.

45 P. Hohenberg and W. Kohn, Phys. Rev., 1964, 136, 864-871.

46 G. Kresse and J. Hafner, Phys. Rev. B: Condens. Matter Mater. Phys., 1993, 47.

47 J. P. Perdew, A. Ruzsinszky, G. I. Csonka, O. A. Vydrov, G. E. Scuseria, L. A. Constantin, X. L. Zhou and K. Burke, Phys. Rev. Lett., 2008, 100.

48 P. E. Blochl, Phys. Rev. B: Condens. Matter Mater. Phys., 1994, 50, 17953-17979.

49 G. Kresse and D. Joubert, Phys. Rev. B: Condens. Matter Mater. Phys., 1999, 59.

50 H. J. Monkhorst and J. D. Pack, Phys. Rev. B: Solid State, 1976, 13, 5188-5192.

51 C. Adamo and V. Barone, J. Chem. Phys., 1999, 110, 6158-6170.

52 M. Gajdos, K. Hummer, G. Kresse, J. Furthmuller and F. Bechstedt, Phys. Rev. B: Condens. Matter Mater. Phys., 2006, 73.

53 A. J. Cruz-Cabeza, CrystEngComm, 2012, 14, 6362-6365.

54 A. Delori, E. Suresh and V. R. Pedireddi, Chem. Eur. J., 2008, 14, 6967-6977.

55 A. Delori and W. Jones, CrystEngComm, 2011, 13, 6315-6318.

56 S. E. Krikorian and M. Mahpour, Spectrochim. Acta, Part A, 1973, 29, 1233-1246.

57 J. Seliger and V. Zagar, J. Phys. Chem. A, 2013, 117, 1651-1658.

58 M. Telzhensky and M. Kaftory, Acta Crystallogr., Sect. C: Cryst. Struct. Commun., 2009, 65, O314-O320.

59 P. Vishweshwar, J. A. McMahon, J. A. Bis and M. J. Zaworotko, J. Pharm. Sci., 2006, 95, 499-516.

60 L. R. MacGillivray, J. Org. Chem., 2008, 73, 3311-3317.

61 J. M. Skelton, E. Lora da Silva, R. Crespo-Otero, L. E. Hatcher, P. R. Raithby, S. C. Parker and A. Walsh, Faraday Discuss., 2015, 177, 181-202.

62 K. T. Butler, C. H. Hendon and A. Walsh, J. Am. Chem. Soc., 2014, 136, 2703-2706. 